\title{
Facilitation of discrimination learning-set in squirrel monkeys by colored food stimuli
}

\author{
HARRIET J. SMITH, JAMES E. KING, and PAUL NEWBERRY \\ University of Arizona, Tucson, Arizona 85721
}

\begin{abstract}
Two groups of squirrel monkeys were trained on discrimination learning-set problems in Phase I. The experimental group discriminated between differently colored edible stimuli which were also reinforcers. In each problem, one colored stimulus tasted sweet and one colored stimulus tasted bitter. Control subjects discriminated between differently colored stimulus blocks upon which identically colored edible reinforcers were placed. In Phase II, both groups were trained on the control task. During Phase I, experimental subjects evidenced significant learning while the control group did not. In Phase II, the experimental group performed significantly above chance, indicating positive transfer between the experimental and control tasks. The results, besides showing the efficiency of the ingested stimulus procedure, indicated a close association between correct and random responding during learning-set acquisition in primates.
\end{abstract}

In 1953, Jarvik described a highly effective technique with which chimpanzees could be taught simple discrimination problems in a few trials. Jarvik's success resulted from his use of colored bread fragments which were reinforcers as well as discriminanda since the negative, but not the positive, colors were saturated with quinine solution. A question raised by Jarvik's experiment is whether use of such colored food stimuli will facilitate discrimination learning-set (DLS) when successful performance requires considerable interproblem reversal of approach and avoidance responses. Although Jarvik's technique facilitates learning of single discriminations, learning could possibly be impaired if subjects must frequently reverse discriminations learned with this technique. Presentation of DLS by the method of minimal stimuli (Riopelle. 1955) entails frequent interproblem reversal of learned approaches and avoidances since all problems are defined by stimulus pairs from a constant set of only four stimuli.

The purpose of the present study was to determine (a) whether DLS acquisition by squirrel monkeys with the method of minimal stimuli would be enhanced or impaired by the use of discriminanda which were also food reinforcers and (b) whether DLS established by the above procedures would transfer to conventionally presented DLS problems.

\section{METHOD}

Subjects

Subjects were 10 young adult male squirrel monkeys (Saimiri

This paper is based in part upon a thesis submitted by the first author in partial fulfillment of the requirements for the Master of Arts degree at the University of Arizona and was supported by training grant $\mathrm{MH}-11286$ from the United States Public Health Service. Requests for reprints should be sent to James E. King. Department of Psychology. University of Arizona, Tucson. Arizona 85721 . sciureus) whose only prior experimental history involved participation in a study of the effect of $(-) \Delta^{9}$-transtetrahydrocannabinol upon social behavior (Jones, Clark, Consroe, \& Smith, 1974).

\section{Apparatus}

A modified version of the Wisconsin General Test Apparatus (WGTA) with manually operated forward opaque screen and stimulus tray was used for adaptation and testing. Two flat $4.5 \times 4.5 \mathrm{~cm}$ blocks were positioned $18 \mathrm{~cm}$ apart on the stimulus tray. The stimulus tray was baited by placement of a small (approximately $30 \mathrm{mg}$ ) colored cookie on top of each block.

The positive cookies were made from powdered sugar, marshmallow creme, food coloring, and water and were baked under low heat until firm. The negative cookies contained a small amount of quinine sulfate added to the above ingredients $(1 \mathrm{~g}$ added to $100 \mathrm{ml}$ cookie mixture), a procedure which did not alter the appearance of the cookies.

\section{Test Procedure}

At the beginning of each trial, the experimenter slowly raised the forward opaque screen of the WGTA and simultaneously pushed the stimulus tray within reach of the monkey. The monkey responded by retrieving either the positive or negative cookie from one of the blocks. Displacement of a block was not possible, and the response was identical in both experimental and control conditions. A noncorrection technique was used throughout adaptation and testing. Subjects were fed their daily food ration immediately upon completion of daily testing.

\section{Adaptation}

All animals were initially trained to retrieve positive cookies placed on top of the blocks on the stimulus tray. The monkeys were then trained on a color discrimination probiem in which a coral-colored positive cookie was placed on one black block and a white negative cookie was placed on the other black block. The position of the positive cookie was determined by a Gellermann sequence. Twenty trials were presented daily until each subject achieved a criterion of $90 \%$ correct responding for each of 3 consecutive days. The colors of the cookies and the blocks used in the adaptation problem were not used during subsequent testing.

\section{Phase I}

After all animals completed adaptation, they were randomly divided into two groups of five animals each and were given a series of six-trial DLS problems. For the experimental subjects, 
the discriminanda were also the reinforcers. The stimulus tray contained two identical brown blocks; a positive cookie of one color was placed on one block while a negative cookie of another color was placed upon the other block. Within each six-trial problem, the colors of the positive and negative cookies remained the same. The position of the positive cookie varied according to a series in which each of the eight possible threetrial left-right sequences occurred equally often. Pink, yellow, orange, and green cookies were used.

In the control condition, positive and negative cookies were both light green. Four flat $4.5 \times 4.5 \mathrm{~cm}$ blocks were the discriminanda in this condition, and each was painted with a close approximation of one of the colors used for the cookies in the experimental condition. Two blocks were used per problem. Thus, the experimental group retrieved and then ingested the discriminanda while the control group simply touched the discriminanda as they retrieved the reinforcers.

The method of minimal stimuli (Riopelle, 1955) was used to define the positive and negative stimuli for both groups. This procedure entails use of only four stimuli instead of the large number of stimuli usually used in DLS problems. These four stimuli (in this case, colors of blocks or cookies) were used repeatedly and the reward value of each color varied across problems. The combination of the four colors into all possible positive-negative pairs yielded 12 different discrimination problems. The problem sequence never included complete reversals or continuation of discriminations on two successive problems. All other possibilities were, however, presented.

The animals were given six trials on each of six problems for 72 test days. All animals were thus presented a total of 432 DLS problems in this phase. Every color was presented as a positive stimulus and as a negative stimulus three times every 2 days, and each problem type appeared approximately the same number of times. Consecutive trials within one problem followed one another with about a 5 -sec delay, but 30 -sec intervals were interposed between the last trial of a problem and the first trial of the succeeding problem.

\section{Phase II}

In Phase II, the experimental subjects were tested on the control task for 84 problems while the control subjects continued training on the control task for the same number of problems.

\section{RESULTS}

\section{Phase I}

Figure 1 shows the percentage of correct responses made by the experimental and control groups during the 72 test days of Phase I. Subjects who ingested the discriminanda (experimental group) demonstrated DLS performance clearly superior to that of subjects which did not ingest the discriminanda (control group) $[F(1,8)=8.68, p<.05]$. The Groups by Days interaction was significant $[F(2,16)=4.06, p<.05]$, reflecting the experimental subjects' improvement over trials and the control subjects' lack of improvement. Mean percentages of correct responses of the experimental and control groups on Trial 1 during this phase were $52 \%$ and $50 \%$, respectively. Neither of these values differed significantly from chance.

Patterns of responding of all subjects were analyzed with a modified version of Levine's (1965) hypothesis analysis technique. All hypotheses and their

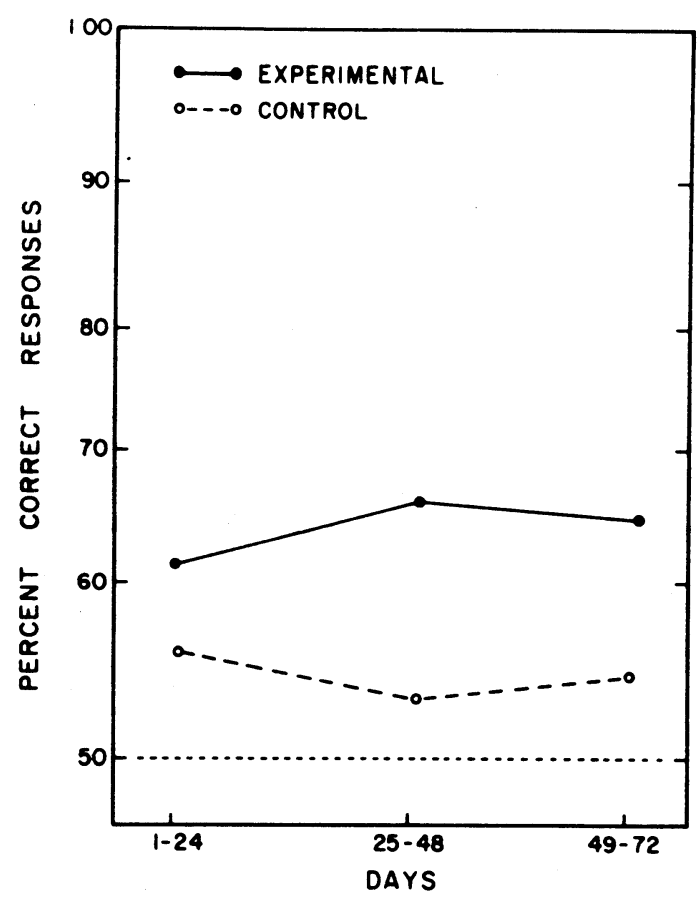

Figure 1. Percentage of correct responses made by experimental and control subjects on Trials 2-6 during Phase II.

behavioral manifestations are presented in Table 1.1 A slightly different set of hypotheses was measured on Trials 1-3 than on Trials 4-6 since Trial 1 but not Trial 4 responding was at chance level.

Figure 2 depicts the strengths of the eight hypothese exhibited by experimental and control subjects on Trials 1-3. Experimental animals showed significantly greater second-trial learning $[F(1,8)=$ 8.85. $\mathrm{p}<.05]$ and significantly less triplet position perseveration than did the control animals $[F(1,8)=6.36, p<.05]$. Third-trial learning, winstay; lose-shift (position), and win-shift; lose-stay (position) hypotheses had negligible strengths for both groups on Trials 1-3.

Figure 2 also depicts the strengths of the nine hypothese exhibited by experimental and control subjects on Trials 4-6. Experimental subjects again achieved significantly greater correct responding $[F(1,8)=5.33, p<.05]$ and significantly less doublet perseveration $[\mathrm{F}(1,8)=9.38, \mathrm{p}<.05)$ than did the control subjects. Second-trial learning, third-trial learning, stimulus preference, win-stay; lose-shift (position), and win-shift; lose-stay (position) hypotheses had negligible strengths for both groups on Trials 4-6.

The interaction between groups and problems was significant for random responding on Trials 4-6 $[F(2,16)=6.54, p<.05]$. Analysis of this interaction indicated that random responding by the experimental group increased nonsignificantly over problems while random responding by the control 
Table 1

Hypotheses in Phases I and II

\begin{tabular}{lll}
\hline \multicolumn{1}{c}{ Hypothesis } & \multicolumn{1}{c}{ Definition } & Manifestations \\
\hline First-trial learning* & Learning manifested on first trial of problem & +++ \\
Second-trial learning & Learning manifested on second trial of problem &,-+++++ \\
Third-trial learning & Learning manifested on third trial of problem &,,,++++++-+--+ \\
Stimulus preference & Sequence of responses to the S+ or $\mathrm{S}-$ & III \\
Triplet perseveration & Three consecutive responses to one side & III, IIO, IOO \\
Doublet perseveration & Two or more consecutive responses to one side & I+I+I, I+I-O, I-O+O, I-O-I \\
Win-stay; lose-shift, position & Response to position rewarded on previous trial & I+O+I, I+O-O, I-I+O, I-I-I \\
Win-stay; lose-stay, position & Response to position not rewarded on previous trial & All sequences \\
Random & Response not correlated with stimulus or prior outcome & \\
\hline
\end{tabular}

Note. + indicates a correct response, - indicates an incorrect response. I indicates the position selected on the first trial of a threetrial sequence, $O$ indicates the other position.

*Measured on Trials 4-6 only.

group decreased significantly over problems $[\mathrm{F}(2.8)=8.31, \mathrm{p}<.05]$.

\section{Phase II}

During the 14 test days of Phase II, the experimental group achieved $60 \%$ correct responses and the control group achieved $57 \%$ correct responses on Trials 2-6. The difference in performance between the experimental and control groups was not signiticant, but the number of correct responses by both groups was significantly above chance $[\mathrm{t}(4)=9.10, \mathrm{p}<.001$ for the experimental group and $t(4)=8.76, p<.001$ for the control group].

The hypothesis analysis showed that experimental animals exhibited significantly more win-shift; lose-stay (position) responding than control animals $[F(1.8)=7.28, p<.05]$ and that the control group exhibited significantly more win-stay; lose-shift (position) responding than the experimental group $[F(1,8)=11.24, p<.05]$ on Trials $4-6$, but the percentage strengths of both hypotheses were quite low. There were no other group differences in percentage strengths of hypothese in this phase.

The experimental subjects attained $52 \%$ correct responses. and the control subjects attained $51 \%$ correct responses on Trial 1 of Phase II. This difference was not significant nor were the differences between either group mean and chance performance on Trial 1.

\section{DISCUSSION}

The results of this experiment demonstrated that colored food stimuli facilitate acquisition of a minimal stimuli DLS by squirrel monkeys and that this training transfers positively to discriminations in which the discriminative stimuli are touched but not eaten by the monkeys. The facilitation of minimal stimuli DLS by colored food stimuli is consistent with Jarvik's $(1953,1956)$ early studies in which chimpanzees learned simple red-green discriminations more rapidly when bread reinforcers contained the discriminative stimuli than when the discriminative stimuli were beside the reinforcers. Allowing the subject to consume discriminative stimuli appears to facilitate discrimination learning including acquisition of single discriminations as well as acquisition of minimal stimuli DLS.

Spatial discontiguities between stimulus and response, response and reinforcer. and stimulus and reinforcer are effective inhibitors of discrimination learning (Miller \& Murphy, 1964; Murphy \& Miller, 1955, 1958). In both treatments of the study described here, stimulus-response-reinforcer discontiguities were virtually zero and therefore probably did not contribute to the different performances of experimental and control subjects. The superiority of the experimental monkeys is probably related to their increased attention to stimuli that are to be eaten.

Although performance of the experimental subjects was significantly above chance during Phase I, it was not high enough to
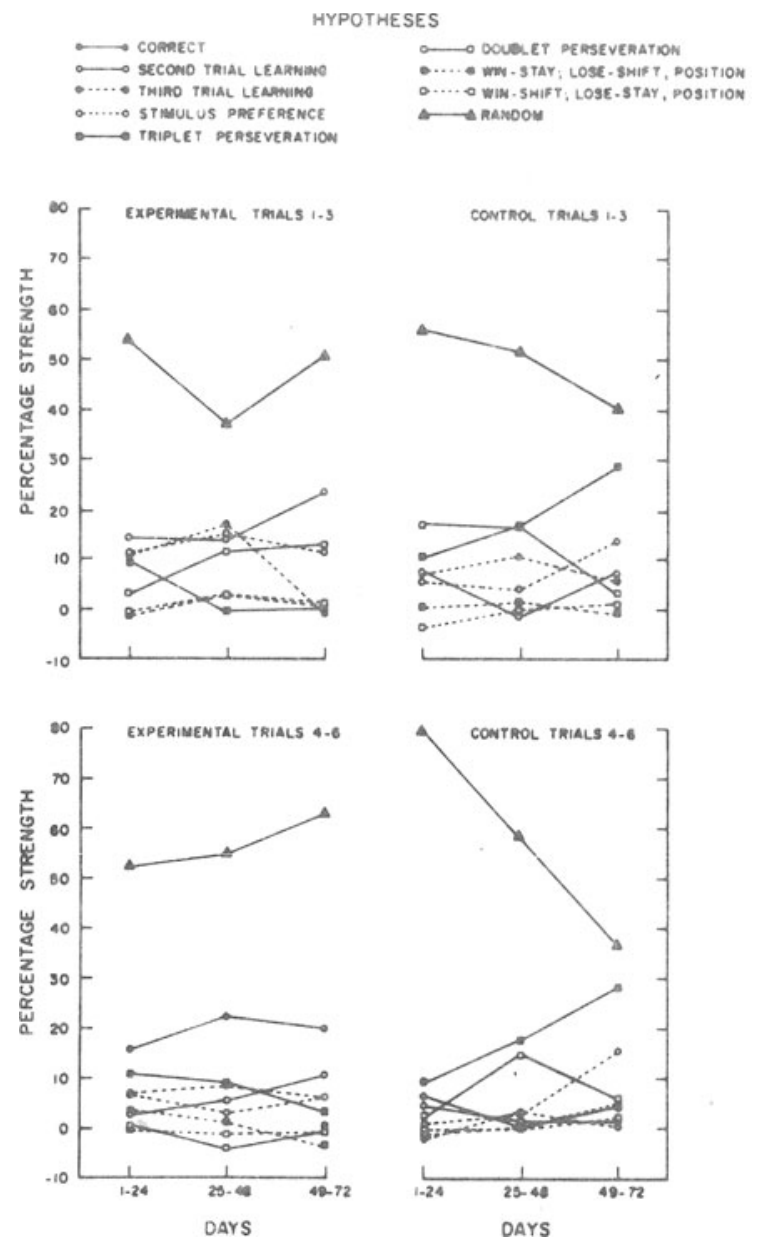

Figure 2. Strengths of various hypotheses during Trials 1-3 and Trials 4-6 for experimental and control subjects. 
signify complete DLS. Two characteristics of the procedure used possibly accounted for the relatively low performance. First, stimulus size is an important variable affecting primate learning of discriminations (Warren, 1953) with large stimuli resulting in faster learning than small stimuli. The stimuli in the experimental condition were small $\left(.28 \mathrm{~cm}^{2}\right)$, which undoubtedly lowered the DLS performance. It is noteworthy that the control group responded to stimuli $20.25 \mathrm{~cm}^{2}$ in area during Phase I and still did not learn. Second, a fixed trials per problem procedure typically leads to slower learning than a criterion procedure on difficult learning tasks such as DLS (Rumbaugh \& McQueeney, 1963).

King (personal communication, February, 1975) used the criterion procedure to train squirrel monkeys on a minimal stimuli DLS task with junk objects and the same apparatus used in the present study. After experiencing the same number of trials as subjects in Phase I, four out of five of King's subjects attained between $50 \%$ and $54 \%$ correct responses on Trials 2-6 of each problem as compared with $65 \%$ correct responses attained by experimental subjects in the present study. That small discriminative stimuli and a fixed trials procedure did not prevent experimental subjects in the present study from acquiring a DLS superior to that in King's experiment is strong evidence of the efficiency of the ingested stimulus procedure.

Application of hypothesis analysis to data from two recent studies of sameness-difference learning-set in capuchin monkeys (King \& Fobes, 1975; Scanlon \& King, Note 1) revealed an interesting difference between fast and slow learners similar to the difference between experimental and control subjects in the study described here. Fast-learning subjects exhibited higher random responding than slow-learning subjects, and the high random responding decreased only when the monkeys began to show a considerable amount of correct responding. Other hypotheses were not strongly evidenced by fast learners, and incidence of position preference was low. Slow learners manifested a greater variety of incorrect hypotheses than fast learners, and their percentage strength of random responding was consequently lower.

These same patterns of hypothesis manifestations were clearly exhibited by the experimental and control group in the present study. Experimental subjects, similar to the fast-learning capuchin monkeys, showed higher correct and random responding than did the control subjects and showed almost negligible position preference. Control subjects, similar to the slowlearning capuchin monkeys, showed high triplet and doublet position preference and relatively high stimulus preference but low correct and random responding. These results indicate a close association between random and correct responding during the early and middle stages of learning-set formation in primates and suggest that random responding may be an index of the subjects' attentiveness to the task. This relationship between random responding and attention is consistent with the previously mentioned suggestion that attention to discriminative cues is enhanced by colored food stimuli.

\section{REFERENCE NOTE}

1. Scanlon. J., \& King, J. E. Transposition along a homogeneity/heterogeneity dimension by capuchin monkeys. Unpublished manuscript. 1975. (Available from author's address.)

\section{REFERENCES}

JARVIK. M. E. Discrimination of colored food and food signs by primates. Journal of Comparative and Physiological Psychologv. 1953, 46, 390-392.

JARVIK. M. E. Simple color discrimination in chimpanzees: Effect of varying contiguity between cue and incentive. Journal of Comparative and Physiological Psychology, 1956, 49, 492-495.

Jones, B. C.. Clark, D. L., Consroe, P. F., \& Smith, H. J. Social behavior of squirrel monkey dyads in a water bottle competition situation. Psychopharmacologia, 1974, 37. 37-43.

KING, J. E., \& FoBEs, J. L. Hypothesis analysis of samenessdifference learning-set by capuchin monkeys. Learning and Motivation. 1975. 6. 101-113.

Levine, M. Hypothesis behavior. In A. M. Schrier, H. F. Harlow, \& E. Stollnitz (Eds.), Behavior of nonhuman primates (Vol. 1). New York: Academic Press. 1965.

Mille R. R. E., \& MURPhY, J. V. Influence of the spatial relationships between the cue, reward, and response in discrimination learning. Journal of Experimental Psychology, 1964, 67, 120-123.

Murphy, J. V..\& Miller, R. E. The effect of spatial contiguity of cue and reward in the object-quality learning of rhesus monkeys. Journal of Comparative and Physiological Psychology, 1955, 48. 221-224.

MurPhy, J. V.. \& Miller, R. E. Effect of the spatial relationship between cue, reward and response in simple discrimination learning. Journal of Experimental Psychology, 1958, 56. 26-30.

Riopelle, A. J. Learning-sets from minimum stimuli. Journal of Experimental Psychology, 1955, 49, 28-32.

Rumbaugh, D. M., \& McQueEney, J. A. Learning-set formation and discrimination reversal: Learning problems to criterion in the squirrel monkey. Journal of Comparative and Physiological Psychology, 1963, 56, 435-439.

WARREN, J. M. The influence of area and arrangement on visual pattern discrimination by monkeys. Journal of Comparative and Physiological Psychology. 1953, 46. 231-236.

\section{NOTE}

1. Since the doublet perseverative hypothesis was defined as one perseverative and one random response on trials two and three of a three-trial block, the possible manifestation defined by three responses to one side (III) was twice as likely as either of the other two possible manifestations, each of which were defined by one alternation of position (100 or II0). The equations for determining the strengths of the hypotheses were appropriately weighted to reflect this inequality. 